\title{
The First Systematic Multi-wavelength Survey of Extragalactic Supernova Remnants
}

\author{
I. Leonidaki ${ }^{1}$, P. Boumis ${ }^{1}$ and A. Zezas ${ }^{2,3,4}$ \\ ${ }^{1}$ Institute of Astronomy, Astrophysics, Space Applications \& Remote Sensing, National \\ Observatory of Athens, I. Metaxa \& Vas. Pavlou St., Palaia Penteli GR-15236 Athens, Greece \\ email: ileonid@noa.gr, ptb@noa.gr \\ ${ }^{2}$ University of Crete, Physics Department \& Institute of Theoretical \& Computational Physics, \\ 71003 Heraklion, Crete, Greece \\ ${ }^{3}$ Foundation for Research and Technology-Hellas, 71110 Heraklion, Crete, Greece \\ ${ }^{4}$ Harvard-Smithsonian Center for Astrophysics, 60 Garden St., Cambridge, MA 02138, USA \\ email: azezas@cfa.harvard.edu, azezas@physics.uoc.gr
}

\begin{abstract}
We present the largest sample of multi-wavelength Supernova Remnants (SNRs) in six nearby galaxies, based on Chandra archival data and deep optical narrow-band $\mathrm{H} \alpha$ and [S II] images as well as spectroscopic observations. We have identified $37 \mathrm{X}$-ray selected thermal SNRs, 30 of which are new identifications and $\sim 400$ optical SNRs, for 67 of which we spectroscopically verified their shock-excited nature. We discuss the properties of the X-ray/optically detected SNRs in different types of galaxies and hence different environments, in order to address their dependence on their Interstellar Medium (ISM). We also discuss the SNR populations in the context of the star formation rate of their host galaxies. We cross-correlate parameters of the optically detected SNRs with parameters of coincident X- ray emitting SNRs in order to understand their evolution and investigate possible selection effects.
\end{abstract}

Keywords. supernova remnants, ISM, star formation, galaxies

\section{Introduction}

Supernova Remnants (SNRs) provide a significant fraction of the mechanical energy that heats, shapes, and chemically enriches the Interstellar Medium (ISM). Therefore, SNRs can yield significant information on the global properties of a galaxy's ISM (Blair \& Long 2004). Furthermore, being the endpoints of core-collapse massive stars $\left(\mathrm{M}>8 \mathrm{M}_{\odot}\right)$ they can be used as proxies for measurements of massive star formation rate (SFR) and studies of stellar evolution (Condon \& Yin 1990).

About 274 SNRs are known to exist in our Galaxy (Green 2009) and a large number of them has been studied in detail (e.g. radio: Green 2009; optical: Boumis et al. 2009, Fesen \& Milisavljevic 2010; X-rays: Reynolds et al. 2009, Slane et al. 2002; infrared: Reach et al. 2006). However, these studies are impeded by Galactic absorption and distance uncertainties, hampering the investigation of SNRs in a wide variety of environments. On the other hand, extragalactic studies of SNRs offer several advantages: we can achieve larger samples in determined distances with much fewer observations, they cover a broader range of metallicities and ISM parameters than our Galaxy while internal Galactic absorption effects are minimized, especially on face-on galaxies.

Detecting large samples of SNRs in a multi-wavelength context can provide several key aspects of the physical processes taking place during their evolution. For example, the blast waves of newly formed SNRs can heat the material behind the shock front to temperatures up to $10^{8} \mathrm{~K}$ producing thermal X-rays. Synchrotron emission in radio 
wavelengths is radiated from the vicinity of the shock as well as from the cooling filaments and is easily detectable throughout the life of the remnant (e.g. Charles \& Seward 1995). Optical filaments are a sign of older SNRs since they form in the cooling regions behind the shock (e.g. Stupar \& Parker 2009). Therefore, multi-wavelength studies can surmount possible selection effects inherent in 'monochromatic' samples of SNRs and provide a more complete picture of their nature and evolution as well as their interplay with the ISM and their correlation with star forming activity.

\section{Sample of Galaxies}

We have embarked in an extensive multi-wavelength investigation of the SNR populations in six nearby galaxies (NGC 2403, NGC 3077, NGC 4214, NGC 4395, NGC 4449 and NGC 5204), involving X-ray and optical data. These galaxies were selected from the Third Catalog of Bright Galaxies (RC3; de Vaucoulers et al. 1995) to fulfill the following criteria: (a) to be late-type ( $\mathrm{T}>4$, Hubble Type); (b) close $(<5 \mathrm{Mpc})$ in order to minimize source confusion; (c) at low inclination $\left(\leqslant 60^{\circ}\right)$ in order to minimize internal extinction and projection effects and (d) above the Galactic plane $\left(|\mathrm{b}|>20^{\circ}\right)$. From this pool of objects drawn from the aforementioned selection criteria, we selected galaxies with Chandra archival data with exposure times long enough to achieve a uniform detection limit of $10^{36} \mathrm{erg} \mathrm{s}^{-1}$.

\section{X-ray SNR investigation}

The first step of this attempt (Leonidaki et al. 2010) was to detect in a self-consistent way X-ray thermal SNRs, that is sources with soft, thermal spectrum $(\mathrm{kT} \leqslant 2 \mathrm{keV})$. For that reason we analyzed Chandra archival data for the six galaxies in our sample. We detected 244 discrete X-ray sources down to a limiting flux of $10^{15} \mathrm{erg} \mathrm{s}^{-1} \mathrm{~cm}^{-2}$. The Xray colors of all the detected sources were calculated and illustrated on plots one for each galaxy (see Leonidaki et al. 2010). On the same plots we added grids for power-law and thermal plasma models for different values of temperature $(\mathrm{kT})$, absorbing $\mathrm{H}$ I column density $\left(\mathrm{N}_{H}\right)$, and photon index $\Gamma$. Sources that appear to have temperatures below 2 $\mathrm{keV}$ and mainly lie on the thermal grid, as well as those which are consistent with the thermal grid within their error bars, are candidate SNRs. In order to verify the thermal or non-thermal emission of these sources, we performed a spectroscopic investigation for all candidate X-ray SNRs with adequate number of counts. Sources with spectra fitted only with thermal plasma model(s) are considered thermal X-ray SNRs. In total, $37 \mathrm{X}$ ray thermal SNRs were identified by this study, 30 of which are new discoveries. Since core-collapse SNRs are the end points of the evolution of the most massive stars, they are good indicators of the current Star Formation Rate (SFR). In order to investigate the X-ray properties of SNRs in different star-forming environments, we correlated the average X-ray luminosity of the SNRs in each galaxy against the total FIR luminosity of their host galaxy. As expected, we do not see any trend between those two properties since the latter is a global indicator. What is intriguing though is that SNRs in irregular galaxies tend to be more luminous than those in spirals. This indicates a difference of the SNR population characteristics between the two samples. We attribute that either to the typically lower metallicities of irregular galaxies than those in spirals or to the higher local densities of the ISM which is often the case in irregular galaxies.

Furthermore, someone would expect a linear relation between the number of X-rayselected SNRs and SFR. To verify this connection, we plot the number of SNRs against the integrated FIR luminosity of each galaxy. A linear correlation coefficient of 0.72 shows that this is a significant correlation. 
In order to examine our results in the context of SNR populations detected in other galaxies, we compare the luminosity distributions of X-ray SNRs in different types of galaxies with the number of X-ray-detected SNRs in the studied sample. Therefore we test if the numbers of SNRs in the irregular galaxies in our sample are consistent with those expected, by simply rescaling the SNR X-ray luminosity Functions (XLFs) of the Magellanic Clouds. We find that the numbers of SNRs in irregular galaxies are more consistent with an MC-like SNR X-ray luminosity function, while those of spiral galaxies are more consistent with the SNR-XLF of the spiral M33.

\section{Optical SNR investigation}

The second attempt of this multiwavelength, extragalactic SNR survey was based on a detailed optical spectro-photometric study (Leonidaki et al. 2013). We obtained optical images through [S II] $6716 \& 6731 \AA$ and $\mathrm{H} \alpha$ filters for the galaxies in our sample, using the $1.3 \mathrm{~m}$ Skinakas (Crete, Greece) telescope. We performed photometry on all detected sources and the SNR classification was based on the well-established criterion of $[\mathrm{S} \mathrm{II}] / \mathrm{H} \alpha>0.4$, pioneered by Mathewson \& Clarke (1973). This study revealed $\sim 400$ photometric SNRs down to a limiting $\mathrm{H} \alpha$ flux of $10^{-15}$ erg $\mathrm{sec}^{-1} \mathrm{~cm}^{-2}$. We extracted long-slit and multi-slit spectra for a large number (134) of photometric SNRs using the $1.3 \mathrm{~m}$ Skinakas (Crete, Greece) and $4 \mathrm{~m}$ Mayall (Arizona, USA) telescopes. Using the aforementioned emission line flux criterion of $[\mathrm{S} \mathrm{II}] / \mathrm{H} \alpha>0.4$ for SNRs, 67 sources spectroscopically verified their shock-excited nature.

We calculated various emission line ratios (e.g. $\log (\mathrm{H} \alpha /([\mathrm{N} \mathrm{II}] 6548,6584 \AA$ A $), \log (\mathrm{H} \alpha /([\mathrm{S}$ II] $6716,6731 \stackrel{\AA}{A})$ and [S II] $(6716) /[\mathrm{S} \mathrm{II}](6731) \AA$ ) of the spectroscopically detected SNRs in order to place them in the diagnostic plots of Sabbadin et al. (1977) and Garcia et al. (1991) (see Leonidaki et al. 2013). These diagrams can help us distinguish the excitation mechanism of the emission lines (photoionization for Hir regions and Planetary Nebulae or collisional excitation for SNRs). The most interesting result at these plots is where the $\mathrm{H} \alpha /[\mathrm{N} \mathrm{II}]$ ratio is present: there is a trend for irregular galaxies to have lower $[\mathrm{N} \mathrm{II}] / \mathrm{H} \alpha$ ratios than those in spirals. Since $[\mathrm{N} \mathrm{II}] / \mathrm{H} \alpha$ is a very sensitive metallicity indicator (more than $[\mathrm{S} \mathrm{II}] / \mathrm{H} \alpha$ ), mainly due to its secondary nucleosynthesis, this indicates a difference of the SNR populations between different types of galaxies due to metallicity.

In order to investigate the optical properties of SNRs in different star-forming environments and derive safe conclusions on their connection with SFR, we plot the number of photometric SNRs against the integrated $\mathrm{H} \alpha$ luminosity of each galaxy. We find a linear correlation coefficient of 0.87 which shows that this is a significant correlation.

Mining for optical SNRs within the six nearby galaxies of our sample revealed 18 sources that are associated with X-ray selected SNRs from Leonidaki et al. (2010). In order to examine whether the optical properties of SNRs are good predictors of X-ray SNRs, we compared their $\mathrm{H} \alpha$ luminosities as well as their $[\mathrm{S} \mathrm{II}] / \mathrm{H} \alpha$ ratios with their X-ray luminosities derived in Leonidaki et al. (2010). However, we do not find any statistically significant correlation between these properties. This indicates the existence of different materials in a wide range of temperatures: the X-ray emission originates from hot material behind the shock front and long cooling timescales while the $\mathrm{H} \alpha$ emission comes from cooling regions of dense recombining gas around the edges of the remnant and short cooling timescales.

In the context of multi-wavelength SNR correlation, we compared the number of optical, X-ray and radio-selected SNRs in the form of Venn diagrams for all galaxies in our sample. This comparison not only provides information on the different evolutionary stages of these sources but also depicts various selection effects. For example, the poor 
match rates between optical and X-ray/radio SNRs could be due to the lack of deep radio surveys for half of our galaxies. The trend of detecting more easily older optical SNRs gives rise to the large difference in the match rate between optical and X-ray SNRs. Furthermore, optical searches are more likely to detect SNRs located in low-diffused regions, while radio/X-ray searches are more likely to detect SNRs in regions of high optical confusion. All these facts highlight the importance of multi-wavelength surveys for the study of extragalactic SNR populations.

\section{Conclusions}

This comprehensive multi-wavelength investigation of SNRs in six nearby galaxies revealed 37 X-ray thermal SNRs, 30 of which are new identifications. In the optical band $\sim 400$ photometric SNRs were detected ( $\sim 350$ of which are new identifications), 67 of which spectroscopically verified their nature. Some of the most interesting results derived from this study are: (a) We find that X-ray selected SNRs in irregular galaxies tend to be more luminous than those in spirals, $\mathrm{i}$ (b) There is a trend of SNRs in irregular galaxies to present lower $[\mathrm{N} \mathrm{II}] / \mathrm{H} \alpha$ ratios than those in spiral galaxies, (c) We do not see a correlation between $\mathrm{L}_{X}-\mathrm{L}_{H \alpha}$ or $\mathrm{L}_{X}-[\mathrm{S} \mathrm{II}] / \mathrm{H} \alpha$, possibly indicating the presence of matter in a wide range of temperatures, (d) There is a linear relation between the number of luminous X-ray/optical SNRs and SFR and (e) There is indication for different luminosity distributions in the SNR populations between spiral and irregular galaxies.

\section{References}

Blair, W. P. \& Long, K. S. 2004, ApJS, 155, 101

Boumis, P., Xilouris, E. M., Alikakos, J., Christopoulou, P. E., Mavromatakis, F., Katsiyannis, A. C., \& Goudis, C. D. 2009, A\& $A, 499,789$

Charles, P. A. \& Seward, F. D. 1995, Exploring the X-ray Universe (Cambridge Univ. Press)

Condon, J. J. \& Yin, Q. F. 1990, ApJ, 357, 97

de Vaucoulers, G., de Vaucoulers, A., Corwin, H. G., Buta, R. J., Paturel, G., \& Fouque, P. 1995, Third Reeference Catalog of Bright Galaxies, Springer, New York

Fesen, R. A. \& Milisavljevic, D. 2010, AJ, 140, 1163

Garcia-Lario, P., Manchado, A., Riera, A., Mampaso, A., \& Pottasch, S. R. 1991, A\&A, 249, 223

Green, D. A. 2009, Bulletin of the Astronomical Society of India, 37, 45. (See: arxiv:0905.3699)

Leonidaki, I., Zezas, A., \& Boumis, P. 2010, ApJ, 725, 842

Leonidaki, I., Boumis, P., \& Zezas, A. 2013, MNRAS, 429, 189

Mathewson, D. S. \& Clarke, J. N. 1973, ApJ, 180, 725

Reach, W. T., Rho, J., Tappe, A., Pannuti, T. G., Brogan, C. L., Churchwell, E. B., Meade, M. R., Babler, B., Indebetouw, R., \& Whitney, B. A. 2006, AJ, 131, 1479

Reynolds, S. P., Borkowski, K. J., Green, D. A., Hwang, U., Harrus, I., \& Petre, R. 2009, ApJ, 695, 149

Sabbadin, F., Minello, S., \& Bianchini, A. 1977, A\&A A, 60, 147

Slane, P., Smith, R. K., Hughes, J. P., \& Petre, R. 2002, ApJ, 564, 284

Stupar, M. \& Parker, Q. A. 2009, MNRAS, 394, 1791

\section{Discussion}

MilisAVLJEviC: Did any of your optical spectra show evidence of broad features indicative of young remnants?

LEONIDAKI: Oxygen rich SNRs with broad emission lines (e.g. [OIII] 5007 A) were excluded from our SNR list since they have different detection techniques. 